\title{
Public Health Bulletin
}

\section{DIRECTIONS FOR THE DECADE}

\section{PLANNING FOR CHANGE}

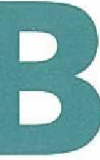
y the year 2001 we will be judging the results of this decade's changes in health service delivery, and their impact on the health status and longevity of the community.

Planning for 2001 and beyond is taking place now within the NSW health system, since there is a long lead-time needed for most changes in health services, especially to physical facilities such as hospitals, or to organisational culture issues such as consumerism.

The latest Corporate Plan for the NSW Health System to be published by the Health Department analyses long-term directions, influences and priorities. It focuses beyond today's operational imperatives on the broad goals and strategies, which in turn inform the other forms of planning and service delivery. The plan gives expression to the vision of the Government and its health managers for the future system.

\section{THE BASIS OF THE PUBLIC SYSTEM}

The NSW health system is focused on increasing the health status of the population, making provision for health care services and ensuring that the community gets value for money.

The public sector health organisations of NSW are part of one of the largest industries in Australia in terms of expenditure and staff employed. The annual budget for $1991-92$ is $\$ 4.25$ billion of public money and there are about 76,000 staff, of whom about 43 per cent are nurses.

Organisations within the public health system include community health centres, hospitals, ambulance stations, rural multi-purpose services, and behind-the-scenes groups such as scientific laboratories and health professional registration boards.

The services delivered by the public health system are complemented by those from the private sector, through facilities such as private hospitals, nursing homes, medical and day surgery centres, and through the services of general practitioners, pharmacists, dentists and other health professionals. More than 5000 Visiting Medical Officers hold appointments in public hospitals, and a number of private contractors supply public hospitals with linen, casual nursing staff and other services.

On an average day in NSW:

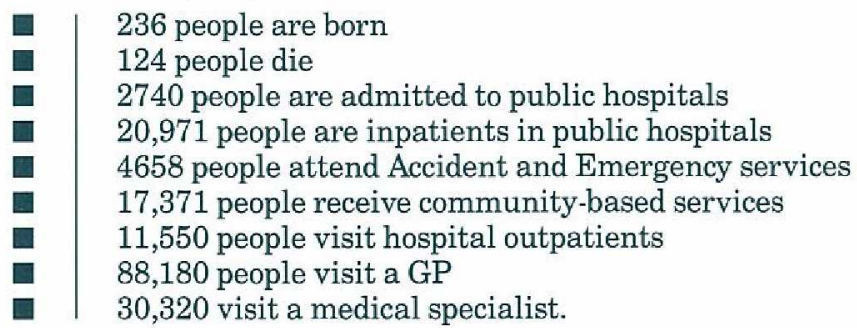

HEALTH STATUS

The health of the people of NSW is very good by world standards and life

\section{Contents}

\section{Articles}

126 Directions for the decade

128 Are co-payments $a$ bealth issue?

129 Travel vaccination beadaches

131 Apparent outbreak of gastrointestinal disease

133 Listeriosis in western Sydney

135 Public bealth abstracts

Infectious diseases

\section{Notifications}

\section{Correspondence}

Please address all correspondence and potential contributions to:

\section{The Editor,}

NSW Public Health Bulletin, Public Health Division, Department of Health, NSW Locked Bag No 961, North Sydney NSW 2059 Telephone: (02) 3919219 Facsimile: (02) 3919232 


\section{Directions for the decade}

\section{Continued from page 126}

expectancy for both males and females ranks with the highest internationally. The health of the Aboriginal community, however, remains a matter for concern and the health problems of some groups of women and migrants continue to require special attention.

Together with most developed countries, Australia's major health problems are increasingly associated with ageing and lifestyle-related conditions - and are potentially amenable to health education.

\section{TECHNOLOGY}

Demands for high technology services compete against the other areas of health care. Decisions about the allocation of resources are often choices between different levels of technology, and also choices between performing simpler services for many people (eg artificial hip joints), or complex services for a few (eg coronary bypass).

Despite the advent of new technologies, the bulk of the work of public hospitals over the next decade is expected to remain in treating commonplace conditions, especially those associated with ageing. The major differences will be the speed with which these conditions can be treated, the introduction of less invasive procedures, the reduction in the need for a lengthy inpatient stay for many conditions, and new treatments for formerly untreatable conditions.

\section{HOSPITALS AND BEDS}

Average lengths of stay in acute hospitals are declining steadily both in Australia and overseas, as a consequence of improvements in medical technology and techniques. The role of principal and major referral hospitals is becoming more focused on the provision of high technology services. Many small rural hospitals are finding they need to provide a broader mix of services, especially long-term care facilities. Continuation of these trends will result in fewer, larger hospitals in the future, with concentration of hospital care on those who are acutely ill. People will find more procedures available on a day-only admission basis, and community-based services will be available to support them at home during their treatment. As the population ages, there will be greater focus on the care of the aged through the provision of specialised community health services and appropriate residential care.

By the year 2001 the average length of stay in hospital is predicted to be 3.64 days (down from the current average of 5.34 days); and the need for acute beds predicted as a total of 23,000 for NSW (down from the present 27,000 public and private beds).

\section{DISTRIBUTION OF RESOURCES}

The distribution of major health facilities is no longer consistent with the spread of the population in NSW.

The Health Department uses a strategy for the redistribution of resources, known as the Resource Allocation Formula. It will result in more equitable distribution of resources by the year 2000 . The formula is based on local population, weighted for a variety of factors such as health status, and is based on the principle that residents should have to travel outside their local area only for highly specialised services.

\section{PRIVATE SECTOR PARTICIPATION}

The private health sector provides most of the primary care and long-term care services, but the public health system provides the majority of acute care beds for both secondary and tertiary services. Over the coming decade the market share for secondary services in the public health system is expected to decline, with the private sector increasing both the volume and size of its facilities.

The maintenance of existing hospital infrastructure continues to take up most funding for capital works. At least 70 per cent of available funds each year needs to be spent on upgrading existing facilities either to expand their capacity or to improve their efficiency and maintain their useful life. This restriction on the use of capital funds for new projects slows the application of the Resource Allocation Formula, and has encouraged negotiations with the private sector on infrastructure provision.

\section{COSTS OF THE PUBLIC HEALTH SYSTEM}

The issue of increasing costs is a complex one. Overall the total proportional cost to the State for the health system has remained stable in recent years, although in dollar terms it has risen substantially. This increase is in line with the increase in GDP and the overall increase in the State's budget, and with the increase in the population.

The demand for increased health care continues to rise faster than the increase in the budget, as new technologies and techniques are developed. However, this rising demand for access (as measured through the waiting times for admission to a public hospital for elective surgery) has been met so far through increases in performance of the system, in terms of greater productivity. This improvement in efficiency is expected to continue over the coming decade.

\section{KEY RESULT AREAS}

The Department has identified a number of key result areas by which the system should be judged over the coming decade. These identify the results sought in relation to both the health status of the population and of the performance of the system. Performance indicators for the system are identified in the key result areas of capacity, productivity, activity, efficiency, workforce and financial performance. Even more importantly, targets are being set for health outcomes, especially in the four priority areas of:

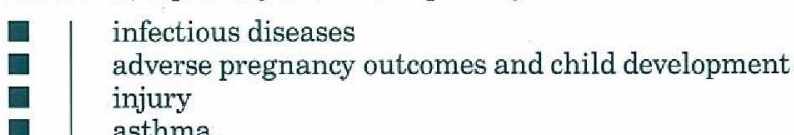

Other conditions targeted are cancers, cardiovascular diseases, malignant melanoma, hip fractures in the aged, diabetes and disabilities.

\section{STRATEGIC DIRECTIONS}

The principal challenge facing NSW is to develop and implement strategies which will improve outcomes and contain the cost of services. The overall development of the future health system is based on the following key strategic themes:

health outcomes improvement and management,
leading the integrated development of the system;
incorporation of the benefits of a national approach;
major structural and technological changes to
service delivery infrastructure;
organisational structure and culture changes to
improve efficiency and effectiveness of services;
long-term financial strategies to facilitate change
and service development; and
advanced information technology to provide
integrated systems for clinical operations and
performance management.

Meryl Edwards,

Corporate Planning Branch

NSW Health Department 